\title{
Wet purpura in Dengue fever
}

\author{
Robin George Manappallil', MounikaYelisetti ${ }^{2}$ \\ ${ }^{1}$ Consultant-Physician, ${ }^{2}$ Resident, Department of Internal Medicine, Baby Memorial Hospital, Calicut, Kerala, India
}

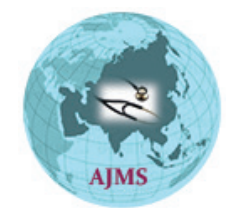

\section{A B S T R A C T}

Dengue fever is an acute febrile viral illness associated with severe headache, myalgia, arthralgia, rashes, leucopenia and thrombocytopenia. These patients can present with haemorrhagic manifestations. Wet purpura in oral mucosa occurs when there is a drastic fall in platelet counts; and can be taken as a sign of impending systemic haemorrhage. This is the case of a young male who was diagnosed to have Dengue fever. He developed severe thrombocytopenia and wet purpura, following which he had gastrointestinal bleed. Dengue fever presenting with wet purpura is a rare scenario.

Access this article online Website:

http://nepjol.info/index.php/AJMS

DOI: 10.3126/ajms.v9i2.19001

E-ISSN: 2091-0576

P-ISSN: 2467-9100

Key words: Wet purpura, Dengue fever, Thrombocytopenia, Malena

\section{INTRODUCTION}

Dengue fever (DF), according to the WHO, is the most rapidly spreading viral disease in the world transmitted by Aedes mosquitoes. It is an acute febrile illness of 2 to 7 days duration, with two or more associated symptoms like headache, retro-orbital pain, arthralgia/myalgia, rash, haemorrhagic manifestations and leucopenia. Dengue haemorrhagic fever is a serious condition, characterised by bleeding manifestations and plasma leakage. ${ }^{1}$ Wet purpura and blood blisters in oral mucosa are indicators of impending life threatening haemorrhage. ${ }^{2}$

\section{CASE REPORT}

A 24 year old male presented to the Medicine OPD with complaints of fever, headache and myalgia since 5 days. On examination, he was conscious, oriented and febrile $\left(102^{\circ} \mathrm{F}\right)$. Other vitals and systemic examinations were normal. His blood investigations showed thrombocytopenia (platelet $89,000 /$ cumm), leucopenia (2,700/cumm) and elevated liver enzymes (SGOT 205 U/L, SGPT 255 U/L). Dengue NS 1 antigen was reactive. Renal functions, electrolytes, prothrombin time and activated thromboplastin time (apTT) were normal. Malarial smear, Leptospira serology, Weil Felix, viral markers (HIV, HBsAg, anti HCV, HAV $\operatorname{IgM}$ ) and Widal test were negative. ECG, chest $\mathrm{X}$-ray and urine microscopy were also normal. He was managed symptomatically with intravenous fluids, folic acid, pantoprazole and paracetamol. The following day, his platelet count reduced to 52,000/cumm. He became afebrile and did not have any bleeding manifestations. On day 3 of admission, he developed wet purpura in his oral cavity (Figure 1). His platelet counts dropped to 9,000/ cumm and apTT was prolonged (test 48 seconds, control 23 seconds). Blood cultures were sterile. Platelet and fresh frozen plasma (FFP) transfusions were given. The next day, he developed malena. His platelet counts were 15,000/ cumm and apTT was mildly prolonged (test 39 seconds, control 20 seconds). Platelet and FFP transfusions were repeated. His vitals continued to be stable. By day 6 , his platelet counts started rising $(48,000 / \mathrm{cumm})$. The wet purpura lesion disappeared and there were no further episodes of malena. He was discharged on day 10 of admission in a stable condition with a normal complete blood count and liver functions.

\section{DISCUSSION}

DF comprises of 3 phases. The initial febrile phase, of 2 to 7 days duration, is characterized by fever, headache, myalgia and leucopenia. This is followed by the critical phase, where the patients are afebrile and face thrombocytopenia, increase in haematocrit levels and capillary permeability. This phase, which lasts for 24 to 48 hours, is accompanied by complications like shock, 


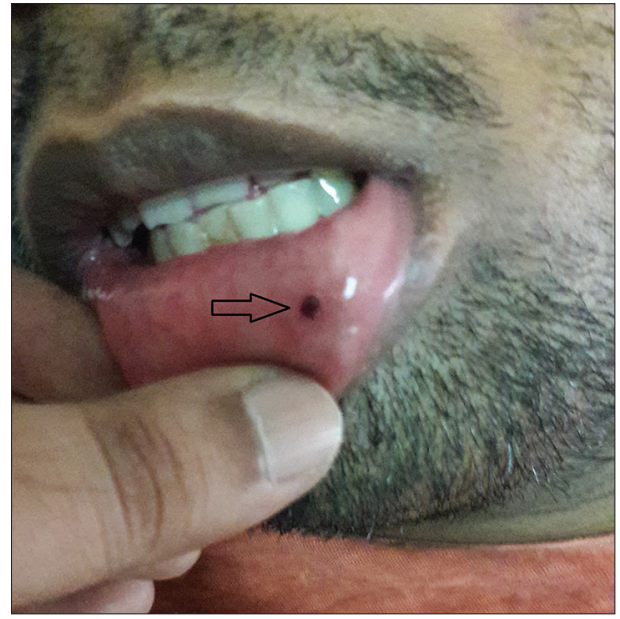

Figure 1: Wet purpura in oral cavity

haemorrhage, myocarditis, hepatitis and encephalitis. Surviving this period, the patients reach the recovery phase, where there is a gradual resorption of the fluid from the extracellular compartment; and the platelet counts show a rising trend with stabilization of haematocrit levels. Intravenous fluids and platelet transfusions are given in hospitalised patients. ${ }^{1}$

A platelet count of 5000 to 10,000 is necessary to maintain the vascular integrity of microcirculation. Petechial patches are seen over the ankles and feet when there is a drastic fall in platelet count. The appearance of wet purpura and blood blisters in oral mucosa are indicators of risk of life threatening haemorrhage. ${ }^{1}$ With regard to DF, prophylactic platelet transfusion may be given at level of $<10,000 /$ cumm, even in absence of haemorrhagic manifestations. Systemic massive bleeding requires platelet and red cell transfusions. FFP or cryoprecipitate are given in case of coagulopathy with bleeding. ${ }^{1}$

Our patient presented during the febrile phase of DF. On entering the critical phase, he had a sudden drop in platelet count, with the appearance of wet purpura in his mouth, and later developed malena. He was managed successfully with platelet and FFP transfusions.

\section{CONCLUSION}

As mentioned earlier, the critical phase of DF is characterized by thrombocytopenia and haemorrhagic manifestations, requiring close monitoring. Wet purpura develops when there is a drastic fall in platelet count; and is a strong indicator of risk of life threatening haemorrhage. The appearance of wet purpura in DF, to the best of our knowledge, is an unreported scenario.

\section{REFERENCES}

1. WHO. Dengue: guidelines for diagnosis, treatment, prevention and control. New edition. Geneva: World Health Organization; 2009.

2. Barbara AK. Disorders of platelets and vessel wall. In: Kasper, Fauci, Hauser, Longo, Jameson, Loscalzo (ed). Harrison's Principles of Internal Medicine. 19th ed. McGraw Hill education. pp. 725-729.

\section{Authors Contribution:}

RGM- Concept and design of case report, reviewed the literature, manuscript preparation and treating Physician; MY- Resident in-charge.

Orcid ID:

Dr. Robin George Manappallil - (1) http://orcid.org/0000-0003-3973-6800

Source of Support: Nil, Conflict of Interest: None declared. 